Rapid Reviews COVID-19

\title{
Reviews of "Vaccination for some childhood diseases may impact the outcome of covid-19 infections"
}

Masako Kinoshita ${ }^{1}$, Jagdish Shukla ${ }^{2}$

${ }^{1}$ National Hospital Organization Utano National Hospital, ${ }^{2}$ University of Montana

Published on: Jan 28, 2022

License: Creative Commons Attribution 4.0 International License (CC-BY 4.0). 
To read the original manuscript, click the link above.

Summary of Reviews: This study reports a negative correlation between vaccination against non-coronavirus infectious diseases and COVID-19 death rates for patients diagnosed in high and low-income countries. Reviewers deemed the study potentially informative, but warn of confounding variables.

Reviewer 1 (Masako Kinoshita)

Reviewer 2 (Jagdish Shukla)

\section{RR:C19 Strength of Evidence Scale Key}

प्रमप = Misleading

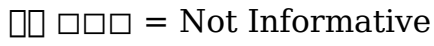

प्रा पि = Potentially Informative

प्र०प्र = Reliable

प्राप्रा = Strong

To read the reviews, click the links below. 